\title{
Elbow flexor and extensor muscle weakness in lateral epicondylalgia
}

\author{
Brooke K Coombes, ${ }^{1}$ Leanne Bisset, ${ }^{2,3}$ Bill Vicenzino ${ }^{1}$
}

1Division of Physiotherapy, School of Health and Rehabilitation Sciences, The University of Queensland, Brisbane, Australia

${ }^{2}$ School of Physiotherapy and Exercise Science, Griffith University, Gold Coast, Australia

${ }^{3}$ Gold Coast Health Services District, Gold Coast, Australia

\section{Correspondence to} Bill Vicenzino, Division of Physiotherapy, School of Health and Rehabilitation Sciences, The University of Queensland, Building 84A, St Lucia OLD 4072, Australia; b.vicenzino@uq.edu.au

Accepted 25 May 2011

\begin{abstract}
Objective To evaluate whether deficits of elbow flexor and extensor muscle strength exist in lateral epicondylalgia (LE) in comparison with a healthy control population.

Design Cross-sectional study.

Participants 150 participants with unilateral LE were compared with 54 healthy control participants.

Main outcome measures Maximal isometric elbow flexion and extension strength were measured bilaterally using a purpose-built standing frame such that gripping was avoided.
\end{abstract}

Results The authors found significant side differences in elbow extensor $(-6.54 \mathrm{~N}, 95 \% \mathrm{Cl}-11.43$ to -1.65 , $\mathrm{p}=0.008$, standardised mean difference (SMD) -0.45 ) and flexor muscle strength $(-11.26 \mathrm{~N}, 95 \% \mathrm{Cl}-19.59$ to $-2.94, p=0.009$, SMD -0.46 ) between LE and control groups. Within the LE group, only elbow extensor muscle strength deficits between sides was significant (affected-unaffected: $-2.94 \mathrm{~N}, 95 \% \mathrm{Cl}-5.44$ to -0.44 ).

Conclusion Small significant deficits of elbow extensor and flexor muscle strength exist in the affected arm of unilateral LE in comparison with healthy controls. Notably, comparing elbow strength between the affected and unaffected sides in unilateral epicondylalgia is likely to underestimate these deficits.

Trial Registration Australian New Zealand Clinical Trials Register ACTRN12609000051246.

\section{INTRODUCTION}

Lateral epicondylalgia (LE) is one of the most common elbow problems in athletes, with 14.1\% of tennis players reporting current LE and $39.7 \%$ reporting current or previous problems. ${ }^{1}$ Patients with LE exhibit an impaired ability to perform tasks that require gripping, with reduced grip force and weakness of wrist flexion and extension. $^{2}{ }^{3}$ In addition, deficits of shoulder abduction, and internal and external rotation strength have also been reported. ${ }^{2}$ Surprisingly, strength of the elbow flexors and extensors has not been previously evaluated in this condition, despite its proximity to the source of pain and important biomechanical function in sporting activities. Indeed, it has been estimated that the elbow contributes $21 \%$ of the total kinetic energy during a tennis service motion. ${ }^{4}$

Comparison of strength of the affected extremity with the unaffected arm is a common clinical practice but may not be valid when there might otherwise be a difference between arms (eg, habituation to predominantly unilateral activity as in tennis). Greater dominant elbow muscle strength has been observed in healthy participants, ${ }^{56}$ in the wrist and forearm ${ }^{27}$ and shoulder. ${ }^{2}$ As LE usually affects the dominant arm, strength deficits were compared between sides and with controls.

Our primary aim was to evaluate maximal elbow flexion and extension strength in participants with unilateral LE. A secondary aim was to investigate the relationship of various patient characteristics with elbow strength in the affected arm.

\section{METHODS}

\section{Study population}

One hundred and sixty-five participants with LE meeting the following criteria were recruited to this study: unilateral elbow pain over the lateral epicondyle for longer than 6 weeks and aggravated by a combination of palpation, gripping and resisted wrist and/or finger extension. ${ }^{8}$ Participants were excluded if they had other primary sources of elbow pain such as cervicogenic, radiohumeral or neurological, or experienced recent fractures, corticosteroid injection or physiotherapy treatment. Fifty-four age- and gender-matched healthy participants with no history of LE were recruited. All participants were excluded if they experienced concomitant neck or other arm pain that prevented participation in their usual work or recreational activities or necessitated treatment within the past 6 months. All participants were recruited from the general community through media advertisements. Ethical approval was granted by the institutional review board and informed written consent was obtained from all participants.

\section{Elbow strength}

Isometric elbow extension and flexion strength were measured in standing with the elbow positioned at $90^{\circ}$ flexion and the forearm in neutral rotation. ${ }^{5}$ Isometric force was measured at the level of the ulna styloid process in Newton using a digital dynamometer (Chatillon; Amtek Lloyd Instruments, Leicester, UK) attached to a purpose-built apparatus. Following a submaximal effort to familiarise with testing, three maximal voluntary isometric contractions were performed, with the maximum recording used in the analysis. The starting test (elbow flexion or extension) was alternated for consecutively recruited study participants and commenced on the unaffected arm (LE) or left arm (controls) and alternating sides with 30 -s intervals. Participants were issued standard verbal encouragement to gradually build up strength and sustain force until a plateau in peak force was reached. Participants were instructed to keep their wrist and fingers relaxed during testing and avoid trunk or scapular movement. The 
investigator observed for any extraneous movement and provided verbal cuing, if correction was needed. Pain intensity was recorded before and during testing on a $100 \mathrm{~mm}$ visual analogue scale (VAS) and testing ceased if pain levels greater than $50 \mathrm{~mm}$ were reported.

To evaluate the test-retest reliability of maximal elbow extension and flexion strength, 10 healthy participants (20 elbows) were tested on two occasions with a 1-week interval by the same assessor. Intraclass correlation coefficients (ICCs) and standard error of the measures (SEMs) were calculated. The $\mathrm{ICC}_{3,1}$ for both extension and flexion were 0.991, and SEMs were 0.75 and 1.13 for elbow extension and flexion, respectively, indicating substantial reliability. ${ }^{9}$ Using the SEM, an estimation of the minimum difference in each measure that must be exceeded to demonstrate true change, as opposed to random measurement fluctuation, was also calculated. ${ }^{10}$ For elbow extension and flexion, we can be $95 \%$ confident that differences greater than 2.09 and $3.14 \mathrm{~N}$, respectively, represent true change. The reliability (ICC $>0.97$ ) and validity of painfree grip (PFG) strength have been previously confirmed. ${ }^{11-13}$

\section{Pain-free grip}

PFG strength was measured bilaterally in supine lying with the elbow positioned in a relaxed extended and pronated position using a digital grip dynamometer (MIE Medical Research, Leeds, UK). ${ }^{14}$ A variable handle position $(25-40 \mathrm{~mm})$ was adjusted such that the maximum distance between a participant's thumb and fingertips when grasping the device was not more than $2 \mathrm{~cm}$. Participants were instructed to grip smoothly and to stop squeezing the dynamometer when their pain was first provoked. Three repetitions were performed, commencing on the unaffected side, with 30 -s intervals. The mean of the repetitions was used in the analysis. ${ }^{12} 15$

\section{Other participant characteristics}

The following demographic characteristics were collected from all participants: gender, age, body mass index (BMI), employment type (manual or non-manual/unemployed) and hand dominance. LE participants were interviewed regarding injury characteristics including injury cause, symptom duration and previous history of LE. Pain intensity (resting pain and worst pain over the preceding week) was measured using $100 \mathrm{~mm}$ VAS. The patient-rated tennis elbow evaluation (PRTEE) was used as a validated measure of pain and functional disability. ${ }^{16} 17$ Possible total scores ranged from 0 (no pain or functional disability) to 100 (worst imaginable pain and very significant functional disability), with pain and function subscales contributing equally.

\section{Data management and analysis}

As hand dominance influences strength in healthy controls, ${ }^{7} 1819$ the proportion of dominant affected elbows in the LE group was replicated in the control group. To achieve this, the control group was randomly allocated a matched affected arm, based on the proportion of dominant, affected arms in the LE group. ${ }^{20}$ Sensitivity analysis of the random allocation of matched upper limbs was performed in order to ascertain any bias due to the matching procedure. From hereon, the matched sides of the control group will also be referred to as affected and unaffected sides.

Analysis of covariance (ANCOVA) was performed using SPSS 19 (IBM, New York, USA) for each strength measure using side (affected vs unaffected) as a within-subject factor and group (LE vs controls) as a between-subject factor. Age, gender and BMI were included as covariates, because of their known effect on strength or significant differences between groups. Interaction plots were inspected in order to understand interaction effects. Follow-up analysis of group effects was performed using univariate ANCOVA with age, gender and BMI as covariates and the differences between sides (calculated by subtracting the unaffected from the affected side) as the dependent variable. $\alpha$ was set at 0.05 . Data are presented as means and $\mathrm{SD}$, mean differences $(\mathrm{MD})$ or standardised mean differences (SMD) and their 95\% CIs. SMD scores were interpreted as small $0.2-0.5$, medium $0.5-0.8$ and large $>0.8{ }^{21}$ Spearman rank coefficients $\left(\mathrm{S}_{\text {rho }}\right)$ were used to evaluate relationships between strength measures and injury characteristics in the LE group. Demographic characteristics were compared between groups using independent $t$ tests.

\section{RESULTS}

Characteristics of the study participants (table 1) were comparable between groups and with previous studies of LE. ${ }^{14} 2223$ Only BMI differed significantly between groups, the LE group being more obese. The LE group comprised middle-aged males and females, predominantly right-handed individuals, with the condition predominantly affecting their dominant arm. They reported a mean 6-month duration of symptoms with $23.3 \%$ reporting previous LE. Most commonly, the onset was reported as insidious (26.7\%), or related to sport (25.3\%), unusual activities $(24 \%)$ and work $(20 \%)$. The mean resting and worst pain levels (VAS) were 9.5 and $60.6 \mathrm{~mm}$, respectively. Thirty-four per cent were engaged in sports involving gripping of a club or racquet while $24 \%$ were employed in manual occupations.

\section{Elbow strength}

Fifteen LE participants were unable to complete elbow strength testing due to pain levels before or during testing. These participants reported significantly greater resting pain (VAS) (MD -14.7 mm, 95\% CI -27.8 to $-1.6, \mathrm{p}=0.03)$, greater total pain and disability (PRTEE) (MD $-17.7,95 \%$ CI -24.7 to $-10.6, p=0.000)$ and weaker PFG in their affected arm (MD $27.4 \mathrm{~N}, 95 \%$ CI 1.2 to $53.5, \mathrm{p}=0.04$ ) than the LE participants who completed testing.

The results of ANCOVA analyses demonstrated significant side by group interaction effects for both maximal elbow

Table 1 Mean \pm SD or count (\%) descriptive data for lateral epicondylalgia $(n=150)$ and control groups $(n=54)$

\begin{tabular}{lcc}
\hline & Lateral epicondylalgia & Controls \\
\hline Demographic characteristics & & \\
Age (years) & $49.7 \pm 8.2$ & $50.1 \pm 10.1$ \\
Females (\%) & $56(37.3 \%)$ & $20(37.0 \%)$ \\
Right arm dominant $(\%)$ & $131(87.3 \%)$ & $48(88.9 \%)$ \\
Body mass index $\left(\mathrm{kg}^{2} / \mathrm{m}\right)$ & $26.8 \pm 4.9 *$ & $25.6 \pm 3.6$ \\
Manual occupation (\%) & $36(24.0 \%)$ & $10(18.5 \%)$ \\
Sport involving gripping (\%) & $51(34.0 \%)$ & $13(24.1 \%)$ \\
Postmenopausal (\% females) & $21(38.2 \%)$ & $12(60.0 \%)$ \\
Injury-related characteristics & & \\
Dominant arm affected (\%) & $109(72.7 \%)$ & \\
Symptom duration (weeks) & $25.6 \pm 31.0$ & \\
Pain at rest VAS (mm) & $9.5 \pm 11.2$ & \\
Pain at worst VAS (mm) & $60.6 \pm 18.2$ & \\
PRTEE (/100) & $37.9 \pm 12.8$ & \\
\hline
\end{tabular}

${ }^{*} p<0.05$.

PRTEE, patient-rated tennis elbow evaluation; VAS, visual analogue scale. 
extensor $(p=0.008)$ and flexor $(p=0.009)$ muscle strength. Interaction plots (figure 1) revealed that elbow strength of the affected arm of LE was weaker than that of the matched arm of controls, while the unaffected arm was comparable with controls. Group by side data are presented in table 2. Follow-up testing of the interaction effects identified significant differences between LE and controls for their between-side differences (elbow extension $(-6.54 \mathrm{~N}, 95 \% \mathrm{CI}-11.43$ to -1.65 , $\mathrm{p}=0.008)$; flexion $(-11.26 \mathrm{~N}, 95 \% \mathrm{CI}-19.59$ to $-2.94, \mathrm{p}=0.009)$ ). These differences exceeded the minimum differences required to be $95 \%$ confident of a true change $(2.09 \mathrm{~N}$ for extension and $3.14 \mathrm{~N}$ for flexion). These values represent small-sized effects (SMD: extension -0.45 ; flexion -0.46$).{ }^{21}$

\section{Pain-free grip}

ANCOVA analysis of PFG demonstrated a significant interaction between group and side $(p=0.000)$, with interaction plots highlighting the large deficit in the affected side of LE (figure 2). Table 2 presents group by side data. Follow-up testing of the differences between sides confirmed the observation from the interaction plot, with the magnitude of the difference between LE and control groups (MD: $-230.94 \mathrm{~N}$, $95 \%$ CI -254.2 to -207.8$)$ representing a very large SMD of -3.15 .

\section{Sensitivity analysis}

All analyses were repeated using independently randomised allocations of matched arms to the control group. This did not change study results.

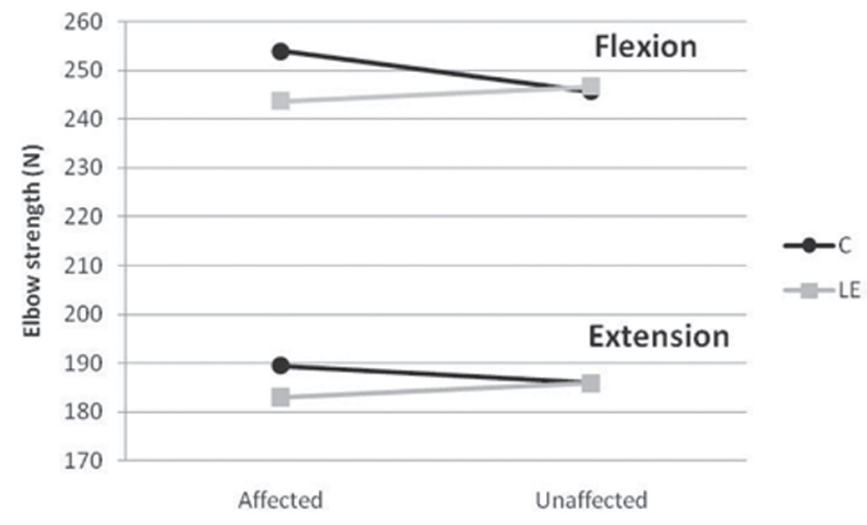

Figure 1 Group (lateral epicondylalgia (LE), control (C)) by side (affected, unaffected) interaction plot for maximal elbow flexion and extension strength. Data are adjusted for age, gender and body mass index.

\section{Pain and disability}

The mean level of elbow pain (VAS) experienced by LE participants during testing of elbow flexion and extension was 8.1 and $7.6 \mathrm{~mm}$, respectively. No correlation was found between pain levels experienced during testing and maximal elbow strength. Pain intensity (VAS) over the preceding week and injury duration did not correlate significantly with any strength measures. There was a weak correlation for PRTEE (pain and disability) ratings with PFG $\left(\mathrm{S}_{\text {rho: }}-0.356, \mathrm{p}=0.000\right)$, elbow flexion $\left(\mathrm{S}_{\mathrm{rho}}-0.217, \mathrm{p}=0.008\right)$ and extension $\left(\mathrm{S}_{\mathrm{rho}}\right.$ $-0.224, p=0.006$ ).

\section{DISCUSSION}

\section{Evidence of elbow flexor and extensor strength deficits}

Our study is the first to document that people with LE exhibit significant deficits of elbow flexor and extensor muscle strength in comparison with healthy controls. These findings are consistent with those from other studies of wrist, forearm, ${ }^{2}{ }^{3}$ and shoulder ${ }^{2}$ muscle strength in LE participants. However, the deficit that we found at the elbow is modest (ie, 6.54 $\mathrm{N}$ for elbow extension and $11.26 \mathrm{~N}$ for elbow flexion) and appear less at the elbow than at the shoulder or wrist. A previous study of $32 \mathrm{LE}$ and healthy control participants reported average deficits of $25-35 \%$ for grip strength, wrist and shoulder strength. ${ }^{2}$

Notably in our study, the dominant arm was affected in the majority $(72.7 \%)$ of LE participants, such that comparison between sides was found to underestimate elbow extensor deficits and fail to detect elbow flexor deficits due to a loss

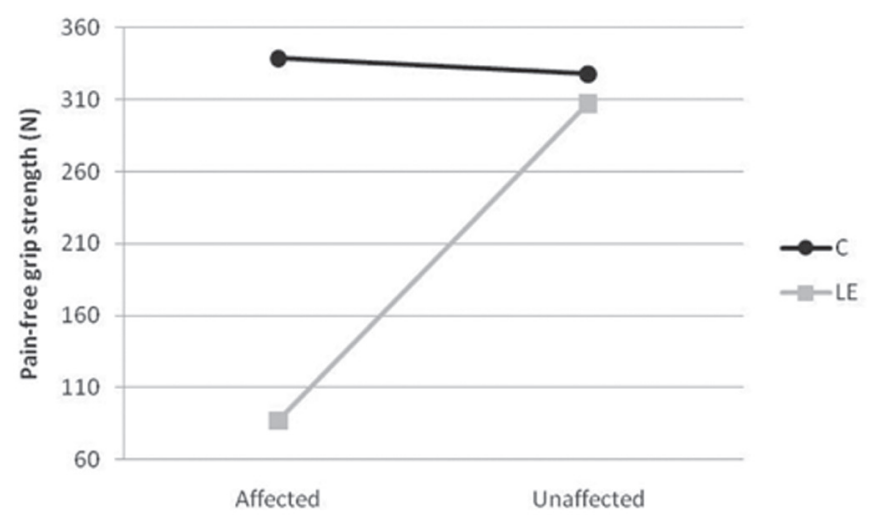

Figure 2 Group (lateral epicondylalgia (LE), control (C)) by side (affected, unaffected) interaction plot for grip strength. Data are adjusted for age, gender and body mass index.

Table 2 Mean, SD and 95\% Cls for strength measures, mean side differences and mean difference (MD) between lateral epicondylalgia (LE) and control (C) groups for side differences (significance represented by ${ }^{\dagger}$ )

\begin{tabular}{|c|c|c|c|c|c|c|}
\hline & & Affected & Unaffected & Side difference* & LE versus C: Side difference* & \\
\hline & & Mean \pm SD & & Mean $(95 \% \mathrm{CI})$ & MD (95\% Cl) & SMD \\
\hline \multirow[t]{2}{*}{ Extension } & LE & $183 \pm 30.6$ & $185.9 \pm 31.8$ & $-2.94(-5.44 \text { to }-0.44)^{\dagger}$ & $-6.54(-11.43 \text { to }-1.65)^{\dagger}$ & -0.42 \\
\hline & $\mathrm{C}$ & $189.5 \pm 30.9$ & $185.9 \pm 32.3$ & $3.6(-0.59$ to 7.78$)$ & & \\
\hline \multirow[t]{2}{*}{ Flexion } & LE & $243.7 \pm 39.2$ & $246.7 \pm 40.4$ & $-2.99(-7.24$ to 1.27$)$ & $-11.26(-19.59 \text { to }-2.94)^{\dagger}$ & -0.42 \\
\hline & C & $253.9 \pm 39.7$ & $245.7 \pm 40.4$ & $8.29(1.15 \text { to } 15.40)^{\dagger}$ & & \\
\hline \multirow[t]{2}{*}{ PFG } & LE & $87.1 \pm 60.0$ & $307.1 \pm 58.8$ & $-220(-231.86$ to -208.15$)$ & $-230.98(-254.15 \text { to }-207.80)^{\dagger}$ & -3.12 \\
\hline & C & $338.6 \pm 61.0$ & $327.6 \pm 58.8$ & $10.97(-8.86$ to 30.80$)$ & & \\
\hline
\end{tabular}

*Differences between sides were calculated by subtracting the unaffected from the affected arm. The control group matched arms are referred to as affected and unaffected. Data were calculated with the following covariates: age 49.8 years, female 0.37 and body mass index 26.5 .

PFG, pain-free grip; SMD, standardised mean difference. 
of dominance bias in the affected arm. Other authors have similarly described a loss of normal dominance-related differences in the LE population. ${ }^{2} 7$ The implications of our findings are that when clinically assessing an individuals' elbow strength, comparison between sides is likely to underestimate or miss true deficits. Failure to return increased dominant arm strength levels to an athlete following injury may represent incomplete rehabilitation. Based on evidence of weakness at multiple upper limb segments, we recommend strengthening the whole upper limb in rehabilitation of LE, in conjunction with exercises to improve grip capacity.

\section{Mechanisms underlying elbow weakness}

It is interesting to speculate on the mechanisms underlying these findings. Decreased maximal knee extension strength following experimentally induced extensor muscle pain has been found to be attributed to central mechanisms. ${ }^{24}$ In our study, pain scores during elbow strength testing were low and not correlated with elbow strength measures, suggesting that pain inhibition is less likely to be the cause of observed weakness. Fear of movement (re)injury and pain may also limit performance during maximal muscle testing. Patients with more pain may consciously or unconsciously anticipate an increase in pain and protect themselves by performing submaximally. Study of patients with low back pain found those who reported increased psychological distress and a higher level of current pain showed increased inhibition of muscle activity, leading to submaximal performance. ${ }^{25}$ Disuse or physical deconditioning may be either a cause or a result of LE, regardless of which might provide a perpetuating factor for chronicity. Altered use of the affected arm as a result of elbow pain could account for disuse-related changes found throughout the whole upper limb.

We found elbow and grip strength to be negatively correlated with the PRTEE scale, that is, greater weakness was evident in LE participants with greater pain and disability. However, the size of these associations were weak, indicating that little of the variation in a patient's pain and disability scores could be explained by elbow and grip strength. This may reflect the complexity of ways in which motor function interacts with and reflects pain and disability. Based on previous findings of moderate correlations between PFG and pain and function scores (VAS), ${ }^{15}$ it appears that PFG remains the recommended measure for establishing the severity of LE and being sensitive to changes in the condition.

\section{Additional considerations}

Several aspects of this study ought to be considered when interpreting the findings. As this study represents a large cross-sectional study, no information on a causal relationship between strength impairment and LE can be made. Further research is needed to determine the relevance of elbow strength deficits in terms of recovery and return to full sporting function and the effect of exercise interventions or mechanotherapy on upper limb muscle strength and tendon healing. ${ }^{26}$ We evaluated a clinically relevant method of testing maximum voluntary muscle strength at the elbow. It is important to understand that this likely assesses only one aspect of motor function and further study is required in order to fully understand the motor deficits in LE. Future research may wish to control for whether a muscle is indeed fully contracting during the test by superimposition of an electrical stimulus, a technique known as twitch interpolation. ${ }^{27}$

\section{What is already known on this topic}

- Patients with LE exhibit weakness at the shoulder, wrist and hand.

- Greater strength is found on the dominant than non-dominant shoulder, elbow, wrist and hand of healthy participants.

- LE affects the dominant arm in the majority of cases.

\section{What this study adds}

- Patients with unilateral LE demonstrate significant deficits of elbow flexor and extensor muscle strength in comparison with healthy controls.

- Comparison between sides is likely to underestimate the size of the deficits, particularly of elbow flexion.

- Evaluation and rehabilitation of upper limb muscle strength deficits beyond grip strength should be considered in the management of LE.

\section{CONCLUSION}

Deficits of maximal isometric elbow flexor and extensor muscle strength exist in unilateral LE compared with healthy controls. A comparison between sides in patients with LE is likely to underestimate these deficits due to normal dominance-related differences between sides. An evaluation of the elbow in conjunction with testing of the forearm and wrist may be used for performance enhancement, injury prevention and rehabilitation.

Funding National Health \& Medical Research Council Grant \#511238.

Competing interests None.

Ethics approval This study was conducted with the approval of the NHMRC, University of Queensland.

Provenance and peer review Not commissioned; externally peer reviewed.

\section{REFERENCES}

1. Gruchow HW, Pelletier D. An epidemiologic study of tennis elbow. Incidence, recurrence, and effectiveness of prevention strategies. Am J Sports Med 1979:7:234-8.

2. Alizadehkhaiyat $\mathbf{0}$, Fisher AC, Kemp GJ, et al. Upper limb muscle imbalance in tennis elbow: a functional and electromyographic assessment. J Orthop Res 2007;25:1651-7.

3. Slater H, Arendt-Nielsen L, Wright A, et al. Sensory and motor effects of experimental muscle pain in patients with lateral epicondylalgia and controls with delayed onset muscle soreness. Pain 2005;114:118-30.

4. Kibler WB. Clinical biomechanics of the elbow in tennis: implications for evaluation and diagnosis. Med Sci Sports Exerc 1994;26:1203-6.

5. Askew LJ, An KN, Morrey BF, et al. Isometric elbow strength in normal individuals. Clin Orthop Relat Res 1987:261-6.

6. Ellenbecker TS, Roetert EP, Riewald S. Isokinetic profile of wrist and forearm strength in elite female junior tennis players. Br J Sports Med 2006;40:411-14.

7. Strizak AM, Gleim GW, Sapega A, et al. Hand and forearm strength and its relation to tennis. Am J Sports Med 1983;11:234-9.

8. Coombes BK, Bisset L, Connelly LB, et al. Optimising corticosteroid injection for lateral epicondylalgia with the addition of physiotherapy: a protocol for a randomised control trial with placebo comparison. BMC Musculoskelet Disord 2009;10:76.

9. Shrout PE. Measurement reliability and agreement in psychiatry. Stat Methods Med Res 1998;7:301-17.

10. Chesterton LS, Sim J, Wright CC, et al. Interrater reliability of algometry in measuring pressure pain thresholds in healthy humans, using multiple raters. Clin J Pain 2007;23:760-6.

11. Stratford PW, Levy DR. Assessing valid change over time in patients with lateral epicondylitis at the elbow. Clin J Sports Med 1994;4:88-91. 
12. Stratford PW, Norman GR, McIntosh JM. Generalizability of grip strength measurements in patients with tennis elbow. Phys Ther 1989;69:276-81.

13. Smidt N, van der Windt DA, Assendelft WJ, et al. Interobserver reproducibility of the assessment of severity of complaints, grip strength, and pressure pain threshold in patients with lateral epicondylitis. Arch Phys Med Rehabil 2002;83:1145-50.

14. Bisset LM, Russell T, Bradley S, et al. Bilateral sensorimotor abnormalities in unilateral lateral epicondylalgia. Arch Phys Med Rehabil 2006;87:490-5.

15. Stratford PW, Levy DR, Gauldie S, et al. Extensor carpi radialis tendinitis: a validation of selective outcome measures. Physiother Can 1987;39:250-5.

16. Rompe JD, Overend TJ, MacDermid JC. Validation of the Patient-rated Tennis Elbow Evaluation Questionnaire. J Hand Ther 2007:20:3-10; quiz 11.

17. Macdermid J. Update: The Patient-rated Forearm Evaluation Questionnaire is now the Patient-rated Tennis Elbow Evaluation. J Hand Ther 2005;18:407-10.

18. Crosby CA, Wehbé MA, Mawr B. Hand strength: normative values. $J$ Hand Surg Am 1994:19:665-70.

19. Incel NA, Ceceli E, Durukan PB, et al. Grip strength: effect of hand dominance. Singapore Med J 2002;43:234-7.

20. Friedman PJ. Isokinetic peak torque in women with unilateral cumulative trauma disorders and healthy control subjects. Arch Phys Med Rehabil 1998;79:816-19.
21. Cohen J,ed. Statistical Power Analysis for the Behavioral Sciences. Hillsdale, NJ: Lawrence Erlbaum 1988.

22. Stasinopoulos D, Stasinopoulos I, Pantelis M, et al. Comparison of effects of a home exercise programme and a supervised exercise programme for the management of lateral elbow tendinopathy. Br J Sports Med 2010;44:579-83.

23. Bisset L, Smidt N, Van der Windt DA, et al. Conservative treatments for tennis elbow do subgroups of patients respond differently? Rheumatology (Oxford) 2007:46:1601-5.

24. Graven-Nielsen T, Lund H, Arendt-Nielsen L, et al. Inhibition of maximal voluntary contraction force by experimental muscle pain: a centrally mediated mechanism. Muscle Nerve 2002;26:708-12.

25. Verbunt JA, Seelen HA, Vlaeyen JW, et al. Pain-related factors contributing to muscle inhibition in patients with chronic low back pain: an experimental investigation based on superimposed electrical stimulation. Clin J Pain 2005;21:232-40.

26. Khan KM, Scott A. Mechanotherapy: how physical therapists' prescription of exercise promotes tissue repair. Br J Sports Med 2009;43:247-52.

27. Miller TA, Allen GM, Gandevia SC. Muscle force, perceived effort, and voluntary activation of the elbow flexors assessed with sensitive twitch interpolation in fibromyalgia. J Rheumatol 1996;23:1621-7. 


\section{BJSM}

\section{Elbow flexor and extensor muscle weakness in lateral epicondylalgia}

Brooke K Coombes, Leanne Bisset and Bill Vicenzino

Br J Sports Med published online June 27, 2011

doi: 10.1136/bjsm.2011.083949

Updated information and services can be found at:

http://bjsm.bmj.com/content/early/2011/06/27/bjsm.2011.083949.full.html

\section{These include:}

References This article cites 25 articles, 8 of which can be accessed free at: http://bjsm.bmj.com/content/early/2011/06/27/bjsm.2011.083949.full.html\#ref-list-1

$\mathbf{P}<\mathbf{P} \quad$ Published online June 27, 2011 in advance of the print journal.

Email alerting Receive free email alerts when new articles cite this article. Sign up in service the box at the top right corner of the online article.

\section{Topic Articles on similar topics can be found in the following collections \\ Collections \\ Musculoskeletal syndromes (273 articles)}

Notes

Advance online articles have been peer reviewed, accepted for publication, edited and typeset, but have not not yet appeared in the paper journal. Advance online articles are citable and establish publication priority; they are indexed by PubMed from initial publication. Citations to Advance online articles must include the digital object identifier (DOIs) and date of initial publication.

To request permissions go to:

http://group.bmj.com/group/rights-licensing/permissions

To order reprints go to:

http://journals.bmj.com/cgi/reprintform

To subscribe to BMJ go to:

http://group.bmj.com/subscribe/ 